\title{
CORRECTION
}

\section{Correction to: Visual acuity correlates with multimodal imaging- based categories of central serous chorioretinopathy}

\author{
Supriya Arora, Dmitrii S. Maltsev (iD, Niroj Kumar Sahoo (D), Deepika C. Parameshwarappa, Claudio lovino (iD, Tarun Arora, \\ Alexei N. Kulikov, Filippo Tatti, Ramesh Venkatesh (D), Nikitha Gurram Reddy, Ram Snehith Pulipaka, Sumit Randhir Singh, \\ Enrico Peiretti and Jay Chhablani iD \\ (c) The Author(s), under exclusive licence to The Royal College of Ophthalmologists 2021
}

Eye (2022) 36:672; https://doi.org/10.1038/s41433-021-01837-y

Correction to: Eye https://doi.org/10.1038/s41433-021-01788-4, published online 13 October 2021
In this article the author name Ramesh Venkatesh was incorrectly written as Ramesh Vankatesh.

The original article has been corrected. 